\title{
Évolution du régime hydrologique dans le bassin versant du Bouregreg, Maroc
}

\author{
Rajae El Aoula ${ }^{1}$, Gil Mahé ${ }^{2}$, Nadia Mhammdi ${ }^{1}$, Abdellatif Ezzahouani ${ }^{3}$, Ilias Kacimi ${ }^{3}$, and \\ Kenza Khomsi ${ }^{4}$ \\ ${ }^{1}$ Institut Scientifique, centre de recherche GEOPAC, laboratoire de Géophysque et Risques Naturels, \\ Université Mohammed V de Rabat, Rabat, Maroc \\ ${ }^{2}$ UMR HydroSciences Montpellier / IRD, Montpellier, France \\ ${ }^{3}$ Déepartement des Sciences de la Terre, Faculté des sciences, \\ Université Mohammed V de rabat, Rabat 10999, Maroc \\ ${ }^{4}$ Service de la qualité de l'air, Centre national du climat, Direction générale de la météorologie, \\ Boulevard Mohamed 4 Tayeb Naciri, Hay Hassani B.P., 8106 Oasis-Casablanca, Maroc
}

Correspondence: Rajae El Aoula (elaoula.rajae@gmail.com)

Published: 16 November 2021

\begin{abstract}
Résumé. Le Bouregreg est le deuxième fleuve du Maroc. Il draine les eaux du centre du Maroc, du Moyen Atlas à l'Atlantique, qui sont stockées dans la retenue du barrage Sidi Mohamed Ben Abdellah créé en 1974 et situé à proximité de Rabat. Sa fonction exclusive est l'alimentation en eau potable pour les besoins individuels et industriels de 6 millions d'habitants de la bande littorale allant de Kénitra à Casablanca. Toutefois, l'exploitation de cette retenue présente aujourd'hui quelques incertitudes surtout en considérant l'augmentation de la population, la baisse des volumes précipités depuis 1979 et le comblement de la retenue par le transport de sédiments dus à l'érosion des sols. L'objectif de cette étude est de d'écrire l'évolution des pluies et des débits du bassin versant du Bouregreg à partir des séries de pluie et débit sur une période de 36 ans, de 1977 à 2013. Nous avons utilisé̀ les indices pluviométrique et hydrométrique, dans le but de caractériser l'hétérogénéité temporelle de la variabilité climatologique et dégager les grandes tendances des séries de données hydroclimatologiques. L'évolution des indices pluviométriques et hydrométriques met en relief la période déficitaire qu'a connu le Maroc au début des années 80. En effet, une baisse générale des pluies est remarquée dans le bassin versant du Bouregreg depuis 1979, ainsi il existe des relations entre la variabilité des pluies de certaines régions et l'Oscillation Nord Atlantique en 1996. Les méthodes statistiques de détection de ruptures montrent que la période de rupture majeure se trouve durant la deuxième moitié des années 70 et le début des années 80 . Depuis cette date la baisse de la pluviométrie s'est installée de manière significative, et une perturbation du régime hydrologique est observée sur la presque totalité du bassin versant du Bouregreg, même si quelques années de meilleures pluviométries ont été observées à la fin des années 2000.
\end{abstract}

\section{Introduction}

Les précipitations représentent le facteur le plus important du climat tant pour les populations que pour les écosystèmes (Kouassi et al., 2010). Ainsi de nombreuses études portent sur la détection des tendances et des variations dans les longues séries hydroclimatologiques. Cette connaissance est pertinente pour l'élaboration des stratégies d'atténuation et d'adaptation au changement climatique dans les régions affectées. En effet, le Maroc fait partie des pays qui ont souffert économiquement, socialement et écologiquement de la sécheresse au cours des dernières décennies (80 et 90 ) (Tsiourtis, 2001; Singla, 2009). Les systèmes naturels et les activités humaines sont les plus à risque en raison du manque d'approvisionnement régulier en eau au cours de l'année. Ceci est principalement dû à la forte variabilité interannuelle 
et à l'importante saisonnalité des précipitations. Le bassin versant du Bouregreg il est l'un des cinq grands fleuves marocains par son débit et sa taille et il présente la particularité d'être limité à $18 \mathrm{~km}$ de son embouchure par le très grand barrage Sidi Mohamed Ben Abdellah d'un volume supérieur à 1 milliard de $\mathrm{m}^{3}$. Son régime hydroclimatique est influencé par la NAO (North Atlantic Oscillation) et la MO (Mediterranean Oscillation) (Khomsi et al., 2013), avec la baisse des pluies de 1979 (Singla et al., 2010), et suite à une augmentation des évènements extrêmes des températures et des vagues de chaleur (Khomsi et al., 2012, 2016). Les conditions de l'érosion et du transport solide ont été modifiées sur ce bassin. Afin d'approfondir la compréhension du fonctionnement hydroclimatique du bassin versant du Bouregreg, il est judicieux de comparaitre l'évolution des pluies et des débits pour mieux décrire les évolutions climatiques dans la zone d'étude. Les stations utilisées pour ce travail présentent un jeu de données intéressant pour tester l'impact du changement climatique sur le régime hydrologique et connaître la variabilité de la ressource en eau qui serait disponible pour les aménagements en aval de ces stations, suivant une méthode qui a été appliquée partiellement au Maroc par Singla et al. (2010).

\section{Matériels et méthodes}

\subsection{Zone d'étude}

Le bassin versant du Bouregreg (situé entre $5.4-6.8^{\circ} \mathrm{W}$ et $32.8-34^{\circ} \mathrm{N}$ ), occupe la presque totalité du plateau central marocain et s'étend partiellement sur la Wilaya de RabatSalé (Fig. 1), les provinces de Khénifra et de Khémisset. Son réseau hydrographique s'articule autour de deux principales rivières, l'oued Bouregreg et l'oued Grou, qui drainent respectivement une superficie de $4000 \mathrm{~km}$ et de $3600 \mathrm{~km}^{2}$ (Lahlou, 1995). Les apports en eau du bassin sont étroitement liés aux précipitations. Le potentiel moyen en eau du bassin du Bouregreg est évalué à $680 \mathrm{~mm}^{3}$ par an (Khomsi, 2014).

L'oued Bouregreg correspond à la partie nord-est du bassin Bouregreg, il prend naissance dans le massif du Moyen Atlas à l'altitude de $1627 \mathrm{~m}$ au niveau du Jebel Mtourzgane et de Grou (Beaudet, 1969), et se jette dans l'océan Atlantique à l'embouchure séparant les villes de Salé au nord et Rabat au sud, après avoir cheminé vers le littoral atlantique à travers la Meseta. Son chenal suit une direction globale rectiligne orientée SE-NW et s'étend sur une longueur de $240 \mathrm{~km}$. Les 23 derniers kilomètres du cours inférieur du Bouregreg se terminent par un estuaire partiellement aménagé (Lahlou, 1995).

\subsection{Jeu de données}

Les données pluviométriques proviennent de la base SIEREM (Dieulin et al., 2006) et des données récentes sont issues de l'agence hydraulique du Bouregreg et de la Chaouia, sur la période 1977-2013. Les données de débit ont été recueillies auprès de 8 stations de mesure de débit par l'agence hydraulique du Bouregreg et de la Chaouia et couvrent une période allant de 1977 à 2007. Les stations étudiées sont situées dans la moitié amont du bassin, et sont peu influencées par la construction du barrage SMBA.

\subsection{Indices pluviométriques et hydrométriques}

Les indices pluviométriques et hydrométriques permettent de caractériser l'hétérogénéité temporelle de la variabilité climatologique et de dégager les grandes tendances dans les séries hydroclimatologiques. Pour mieux visualiser les périodes de déficit et d'excédent à l'échelle annuelle, ces indices ont été centrés et réduits à partir de la formule suivante :

$\mathrm{Vcr}=(P-\mathrm{MML}) / \sigma$

Où, Vcr est la Variable centrée réduite (Indice pluviométrique (SPI) ou hydrométrique (SDI) selon la variable étudiée), $P$ est le cumul de la variable sur une période $t$ donnée, MML est la moyenne mobile lissée de la série chronologique sur la période d'étude et $\sigma$ est l'écart type de la série sur la période d'étude (Hallouz et al., 2013).

\subsection{Tests statistiques de détection de ruptures}

Ces tests ont pour objectif de détecter une tendance (ou une rupture en moyenne dans les séries. Les tests utilisés dans ce travail sont regroupés dans le logiciel Khronostat (1998) réalisé par l'IRD et disponible sur le site d'HydroSciences Montpellier (http://www.hydrosciences.fr, la date du dernier accès : 2012).

Le test de Pettitt (Pettitt, 1979) a été utilisé sur tous les postes retenus en vue d'identifier une « rupture » moyenne dans les séries chronologiques de pluviométries et hydrométries annuelle et mensuelle étudiées. Ce test réputé pour sa robustesse, est non paramétrique et dérive de la formulation du test de Mann-Whitney. L'absence d'une rupture dans la série chronologique $X$ constitue l'hypothèse nulle $H 0$.

Ensuite, la méthode bayesienne de Lee et Heghinian (1977) permet de déterminer la date et l'amplitude de la rupture. Cette méthode fournit la probabilité qu'un changement se produise à un moment $t$, dans une série chronologique où on suppose à priori qu'il y a effectivement un changement à un moment indéterminé. De même, cette procédure donne une estimation de la probabilité que l'amplitude du changement ait la valeur du changement éventuel de la moyenne.

La segmentation de Hubert proposée par Hubert et al. (1989), consiste à découper la série en $m$ segments $(m>1)$ de telle sorte que la moyenne calculée sur tout segment soit significativement différente de la moyenne du (ou des) segment(s) voisin(s). Une telle méthode est appropriée à la recherche de multiples changements de la moyenne. 


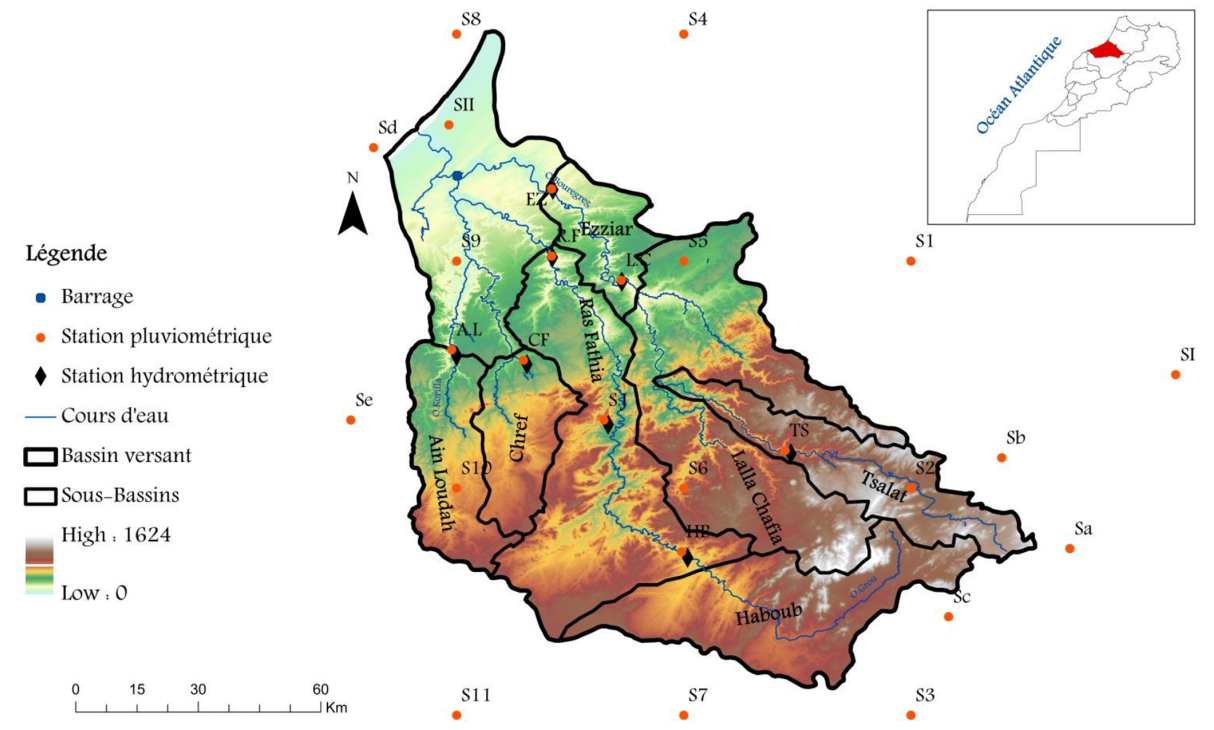

Figure 1. Le bassin versant du Bouregreg et les principaux sous-bassins.

\section{Résultats et discussion}

\section{1 Évolution des relations pluie-débit pour l'ensemble du bassin du Bouregreg}

\subsubsection{Indices pluviométriques et hydrométriques}

Sur la base des indices exprimés par les données pluviométriques et hydrométriques pour la période 1977-2007, on remarque que le graphique des précipitations suit le même sens que celui des débits mais avec des valeurs parfois sensiblement différentes. Cette période est marquée par deux années de déficit pluviométrique 1994 et 2006 (Fig. 2). Après la fin des années 70 on observe une augmentation non seulement de la fréquence des années sèches mais aussi de l'amplitude de la sécheresse par rapport aux deux années précédentes (1978 et 1979). Cette diminution des précipitations est déjà signalée par de nombreux chercheurs dont (Singla et al., 2010) qui ont évoqué la rupture des pluies au Maroc en 1979. Les décennies 80 et 90 sont particulièrement sèches avec le maximum de déficit en 1994. En dehors des années 1995 et 1996 caractéristiques de forte anomalie NAO favorable à la pluie au Maroc (Khomsi et al., 2012), les décennies 80 et 90 sont particulièrement sèches avec le maximum de déficit en 1994.

Sur la Fig. 3 on trace la différence entre l'indice pluviométrique et l'indice hydrométrique. On en déduit une différence positive depuis le début de la sécheresse (1979) jusqu'à l'événement NAO (1995/1996), puis à partir de 1996 la différence devient négative, avec un indice pluviométrique plus fort que l'indice hydrométrique. La diminution brusque enregistrée en 2006 est principalement due à la courte période de disponibilité de données, environ 8 stations régionales pour chaque année. Ce résultat semble être expliqué par le fait que

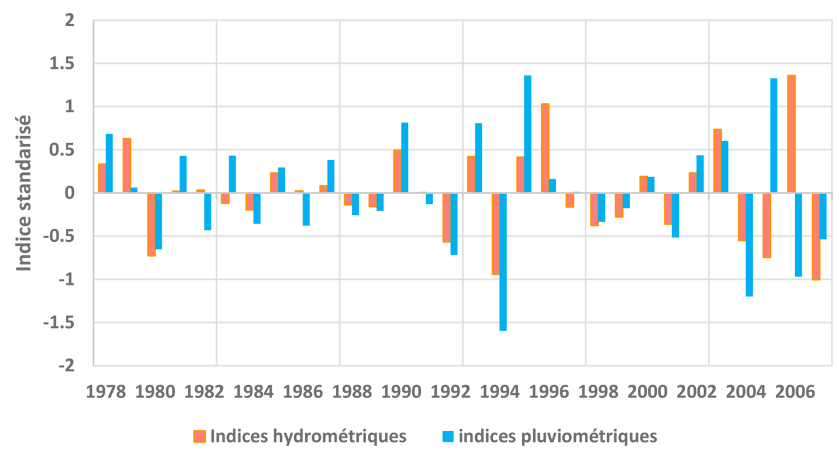

Figure 2. Indices pluviométriques et hydrométriques de 1978 à 2007 pour l'ensemble du bassin versant du Bouregreg $\left(9970 \mathrm{~km}^{2}\right)$.

la variabilité du cumul annuel est plus liée à celle du nombre d'événements plutôt qu'à la quantité de pluie par événement pluvieux (Lawin et al., 2011). Au niveau du bassin versant du Bouregreg, le nombre de jours de pluie par an varie de 60 à 75 entre les montagnes et l'océan et de 75 à 100 sur les régions montagneuses, et les changements dans la variabilité du climat local sont influencés par les changements de la circulation atmosphérique à large échelle.

Khomsi et al. (2013) ont montré l'existence d'un lien statistique significatif entre l'évolution des épisodes chauds d'été et la MO sur les zones internes du Maroc. Une corrélation significative a été également trouvée entre la NAO et les épisodes chauds de Rabat-Salé. En effet, au cours de la saison froide, la NAO est dans sa phase négative, les perturbations s'engouffrent vers le Nord de l'Afrique, apportant ainsi des pluies sur la région. Les types de temps résultants peuvent adoucir le climat et diminuer la fréquence des épisodes chauds au Nord du Maroc. Alors qu'au cours de la 
Tableau 1. Ruptures détectées par les tests de KhronoStat pour les séries de pluies et débits moyennes sur le bassin versant du Bouregreg, période 1977-2007. (NR = Pas de rupture).

\begin{tabular}{|c|c|c|c|c|c|c|c|c|}
\hline \multirow[t]{2}{*}{ Variable } & \multirow[t]{2}{*}{ Période } & \multirow[t]{2}{*}{ Pettitt } & \multirow[t]{2}{*}{ Lee and Heghinian } & \multirow[t]{2}{*}{ Hubert } & \multirow[t]{2}{*}{ Buishand } & \multicolumn{3}{|c|}{ Probabilité de rupture } \\
\hline & & & & & & Faible & Moyen & Fort \\
\hline Pluies & 1977-2007 & 1997 & 1997 & $\begin{array}{l}1994 \\
1998 \\
2006\end{array}$ & NR & - & 1997 & - \\
\hline Débits & 1977-2007 & NR & 1979 & 1979 & 1979 & - & - & 1979 \\
\hline
\end{tabular}

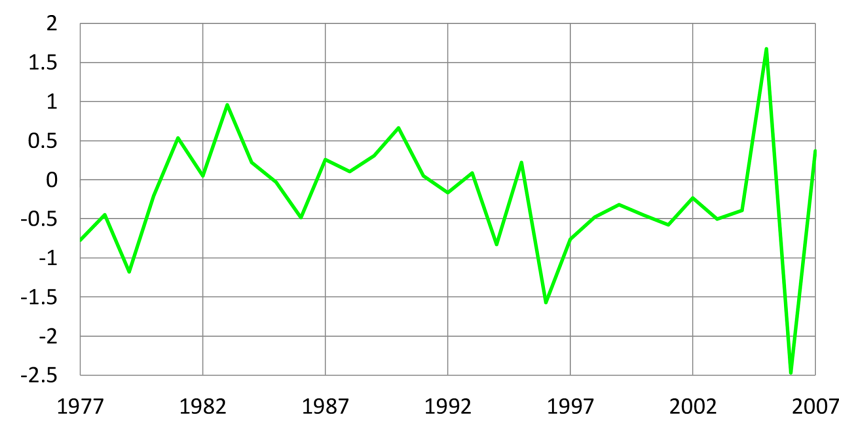

Figure 3. Différence indice pluviométrique moins indice hydrométrique.

saison chaude, la NAO est généralement en phase positive, une augmentation de cet indice renforce les vents d'Ouest sur l'Afrique du Nord. Ces vents devenant plus chauds suite à l'augmentation de la température de la surface de la mer affectent le Nord du pays dont fait partie le Bouregreg. Aussi, l'augmentation de l'indice MO provoque l'infiltration sur le pays, de masses d'air chaudes depuis la Méditerranée. Ces infiltrations peuvent occasionner des épisodes chauds.

\subsubsection{Rupture de stationnarité}

L'analyse et l'interprétation des tests statistiques a permis de constater que pour les pluies annuelles (Tableau 1) la rupture sur l'ensemble du bassin a été détectée au niveau des années 1994 et 1997. Celles-ci correspondent à une diminution des précipitations due à la variabilité climatique. Néanmoins, quelques ruptures et tendances peuvent être observées à la fin de la décennie 70 ou au début de la décennie 80, traduisant peut-être un changement qui se révélerait plus net à l'échelle de l'événement pluvieux. Ces résultats réaffirment les constatations des différentes études réalisées auparavant sur l'évolution des pluies en Afrique comme celles de Klaus (2002), les périodes où le réchauffement est le plus rapide en Afrique au cours du XXe siècle, sont observées à partir des années 1910 jusqu'aux années 1930 et après 1979. Ce qui confirme aussi les études démontrant une diminution des précipitations au Maroc, depuis les années 1960 (Driouech, 2006; DMN, 2007) et plus significativement de- puis la fin des années 70/début des années 80 (Agoumi et Debbarh, 2006; Knippertz et al., 2003). Cette rupture est aussi confirmée à l'échelle régionale, par l'étude de la pluviométrie régionale du bassin versant du Bouregreg (Khomsi, 2014).

Il apparait ainsi qu'un changement du régime pluviométrique et hydrométrique a affecté une grande partie du bassin versant du Bouregreg à partir de 1979.

\subsubsection{Régime mensuel avant et après la rupture (1979)}

Le régime des pluies mensuelles sur les deux périodes avant et après la rupture (Fig. 4) montre une diminution sensible surtout entre décembre et février, estimée à $33 \%$ sur l'ensemble du bassin et dû au changement de régime de pluie interannuel en 1979. On constate que la pluviométrie a baissé au cours des années 80, sauf en mars. Les totaux pluviométriques ont évolué au cours de la «grande » saison des pluies novembre-décembre, tandis que la «petite» saison des pluies de mars à avril a presque disparu, et qu'un maximum relatif apparaît en septembre-octobre. L'examen de la Fig. 3 indique que avant et après la période de la rupture, il constate qu'une diminution significative de $14,3 \%$ de hauteur des précipitations, soit d'un total pluviométrique de 534 à $458 \mathrm{~mm}$, alors qu'au mois de février on note une augmentation de $49 \%$ de lame d'eau tombée. L'impact de cette baisse des pluies est plus important sur les débits qui présentent un changement de régime mensuel très significatif, traduits par une baisse des débits mensuels de printemps, et des débits plus élevés en février et mars sur la période courte 1977-1979. La différence mensuelle de débit entre les deux périodes est de près de $41 \%$.

\section{Conclusion}

L'analyse de l'étude de l'évolution hydroclimatique montrent que le régime pluviométrique général, sur le bassin du Bouregreg, est caractérisé par une baisse des pluies encore accentuée aux alentours de l'année 1979.

Les méthodes statistiques de détection de ruptures ont montré que la période de rupture majeure se trouve durant la deuxième moitié des années 70 et le début des années 80 . 

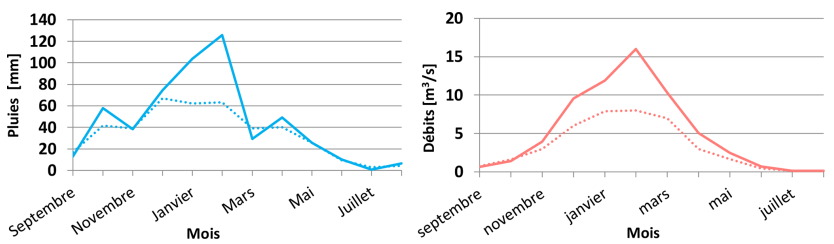

Figure 4. Évolution des pluies et débits mensuels sur le bassin du Bouregreg entre 1977 et 1997 (trait plein) et 1980 à 2013 (tireté) de septembre à août.

Après cette période l'hydraulicité du bassin versant du Bouregreg n'a cessé de se dégrader avec une alternance de périodes humides et sèches au regard de l'évolution des pluies et débits. Cette tendance persistante à la baisse doit être soulignée car elle montre une dégradation durable du système hydrologique malgré un retour assez sensible à de meilleures conditions de précipitation. Ainsi, le régime hydrologique du bassin est directement influencé par le régime de précipitations. Nous supposons donc que la diminution des débits est due principalement à la diminution des précipitations sur le bassin versant du Bouregreg, et non à une baisse majeure des ressources en eau souterraines.

Disponibilité du code et des données. Les données sont disponibles sur demande.

Collaborateurs. REA contributed to the conception and development of this research, data analysis and their interpretation, and drafting text. GM is the supervisor and leadership responsible for this research activity planning and execution. NM participated in the process of preparing this research. AE participated in the data processing and their interpretation. IK participated in the collection of data, and discussions. KK contributed to the preparation and creation of the published work, specifically critical review, commentary and revision- including pre and post-publication stages.

Intérêts concurrents. Les auteurs déclarent qu'ils n'ont aucun conflit d'intérêts.

Clause de non-responsabilité. Publisher's note : Copernicus Publications remains neutral with regard to jurisdictional claims in published maps and institutional affiliations.

Déclaration du numéro spécial. This article is part of the special issue "Hydrology of Large River Basins of Africa". It is a result of the 4th International Conference on the "Hydrology of the Great Rivers of Africa”, Cotonou, Benin, 13-20 November 2021.
Remerciements. Ce travail s'inscrit dans le cadre du projet Toubkal No. TBK/15/CASBA (Caractères Anthropiques et environnementaux de l'érosion des versants du barrage Sidi Mohammed Ben Abdellah) entre le laboratoire Géophysique et Risques Naturels de l'Institut Scientifique, l'agence de bassin hydraulique du Bouregreg et de la Chaouia (ABHBC) et le laboratoire HydroSciences Montpellier.

\section{Références}

Agoumi, A. and Debbarh, A. : Ressource en eau et bassins versants du Maroc : 50 ans de développement (1955-2000), 62 pp., available at : http://www.rdh50.ma/fr/pdf/contributions/GT8-I. pdf (la date du dernier accès : 2018), 2006.

Beaudet, G. : Le plateau central marocain et ses bordures : étude géomorphologique, Thèse, Imprimeries françaises et marocaines, Rabat, p. 478, 1969.

Dieulin, C., Boyer, J. F., Ardoin-Bardin, S., Ardoin-Bardin, S., and Dezetter, A. : The contribution of GIS to hydrological modeling. Climate variability and change : hydrological impacts, FRIEND 2006, edited by : Demuth, S., Gustard, A., Pianos, E., Scatena, F., and Servat, E., Vth Int. Conf., La Havane, Cuba, IAHS Pub., 30868-30874, IAHS Press, Wallingford, UK, 2006.

Direction de la Météorologie Nationale (DMN) : Les changements climatiques au Maroc : Observations et projections, DMN, Secrétariat d'Etat auprès du Ministère de l'Energie, des Mines, de l'Eau et de l'Environnement, Chargé de l'Eau et de l'Environnement, Royaume du Maroc, Casablanca, 2007.

Driouech, F. : Etude des indices de changements climatiques sur le Maroc : températures et précipitations, DMN “INFOMET", Secrétariat d'Etat auprès du Ministère de l'Energie, des Mines, de l'Eau et de I' Environnement, Charge de l'Eau et de l'Environnement, Royaume du Maroc, Casablanca, 2006.

Hallouz, F., Meddi, M., and Mahe, G. : Modification du régime hydroclimatique dans le bassin de l'Oued Mina (nord-ouest d'Algérie), Journal of Water Science, 26, 33-38 2013.

Hubert, P., Carbonnel, J. P., and Chaouche, A. : Segmentation des séries hydrométéorologiques. Application à des séries de précipitations et de débits de l'Afrique de l'Ouest, J. Hydrol., 110, 349-367, 1989.

Khomsi, K. : Variabilité hydroclimatique dans les bassins versants du Bouregreg et du Tensift au Maroc : moyennes, extrêmes et projections climatiques, Thèse de Doctorat, spécialité, Climatologie, Université Mohamed V, Rabat, Maroc, 2014.

Khomsi, K., Mahe, G., Sinan, M., and Snousi, M. : Evolution des évènements chauds rares et très rares dans les bassins versants du Tensift et du Bouregreg (Maroc) et identification des types de 70 temps synoptiques associés, in : From prediction to prevention of hydrological risk in Mediterranean countries, edited by : Ferrari, E. and Versace, P., 4th international Workshop on Hydrological extremes MEDFRIEND group, University of Calabria, EdiBios, Cosenza, Italia, 169-182, 2012.

Khomsi, K., Mahe, G., Sinan, M., and Snousi, M. : Hydroclimatic variability in two Moroccan watersheds : A comparative analysis of temperature, rain and flow regimes. Climate and land surface changes in hydrology, edited by : Boegh, E., Blyth, E., Hannah, D. M., Hisdal, H., Kunstmann, H., Su, B., and Yilmaz, K. K., IAHS Publ. 359, 183-190, 2013. 
Khomsi, K., Mahe, G., Tramblay, Y., Sinan, M., and Snoussi, M. : Regional impacts of global change : seasonal trends in extreme rainfall, run-off and temperature in two contrasting regions of Morocco, Nat. Hazards Earth Syst. Sci., 16, 1079-1090, https://doi.org/10.5194/nhess-16-1079-2016, 2016.

Khronostat : Logiciel de tests statistiques de ruptures dans des séries chronologiques, IRD, France, accessible à l'adresse suivante : http://www.hydrosciences.fr (la date du dernier accès : 2012), 1998.

Klaus, T. : Afrique changements climatiques. Tendance des variations de températures mondiales au cours du XXème siècle, Vital Graphics, p. 4, 2002.

Knippertz,, P., Christophn, M., and Speth, P. : Long term precipitation variability in Morocco and the link to the large-scale circulation in recent and future climates, Met. Atmos. Phys., 83, 67-88, 2003.

Kouassi, A. M., Kouamé, F. K., Koffi, Y. B., Dje B. K., Paturel, E. J., et Oulare, S. : Analyse de la variabilité climatique et de ses influences sur les régimes pluviométriques saisonniers en Afrique de l'Ouest : cas du bassin versant du N'zi (Bandama) en Côte d'Ivoire, CyberGeo, July 2010, 2010.

Lahlou, A. : Etude actualisée de L'envasement des barrages au Maroc, Revue des sciences de l'eau, 6, 337-356, 1995.
Lawin, A. E., Afouda, A., et Lebel, T. : Analyse de la variabilité du régime pluviométrique dans la région agricole d'Ina au Bénin, European Journal of Scientific Research, 50, 425-439, 2011.

Lee, A. F. S. and Heghinian, S. M. : A shift of the mean level in a sequence of independent normal random variables, a Bayesian approach, Technometrics, 19, 503-506, 1977.

Pettitt, A. N. : A non-parametric approach to the change point problem, Appl. Statist., 28, 126-135, 1979.

Singla, S. : Impact du changement climatique global sur les régimes hydroclimatiques au Maroc : tendances, ruptures et effets anthropiques sur les écoulements, Mémoire de Master, M2R2E, France, 2009.

Singla, S., Mahé, G., Dieulin, C. Driouech, F., Milano, M., El Guelai, F. Z., et Ardoin-Bardin, S. : Evolution des relations pluiedébit sur des bassins versants du Maroc, Global Change : Facing Risks and Threats to Water Resources, Proc. of the Sixth World FRIEND Conference, Fez, Morocco, October 2010, IAHS Publ. 340, 679-687, 2010.

Tsiourtis, S. : Drought management plans for the Mediterranean region, Report of the Water Engineer Water Development Department, Nicosia, Cyprus, 2001. 